\title{
Correction to: Highly sensitive and robust peroxidase-like activity of Au-Pt core/shell nanorod-antigen conjugates for measles virus diagnosis
}

Lin Long ${ }^{1,2+}$, Jianbo Liu ${ }^{1 *+} \mathbb{D}$, Kaishun Lu², Tao Zhang ${ }^{2}$, Yunqing Xie ${ }^{2}$, Yinglu $\mathrm{Ji}^{3}$ and Xiaochun $\mathrm{Wu}^{3^{*}}$

\section{Correction to: J Nanobiotechnol (2018) 16:46}

https://doi.org/10.1186/s12951-018-0371-0

After publication of the original article [1], an error was noted in the author affiliation. Lin Long is also affiliated to the College of Opto-electronic Engineering, Zaozhuang University, Zaozhuang, China, which is her first affiliation.

\section{Author details}

${ }^{1}$ College of Opto-electronic Engineering, Zaozhuang University, Zaozhuang 277160, China. ${ }^{2}$ Zaozhuang Municipal Center for Disease Control and Prevention, Zaozhuang 277100, China. ${ }^{3}$ CAS Key Laboratory of Standardization and Measurement for Nanotechnology, National Center for Nanoscience and Technology, Beijing 100190, China.

\section{Publisher's Note}

Springer Nature remains neutral with regard to jurisdictional claims in published maps and institutional affiliations.

Published online: 10 January 2019

\section{Reference}

1. Long L, Liu J, Lu K, Zhang T, Xie Y, Ji Y, Wu X. Highly sensitive and robust peroxidase-like activity of Au-Pt core/shell nanorod-antigen conjugates for measles virus diagnosis. J Nanobiotechnol. 2018;16:46.

\footnotetext{
*Correspondence: linyibm@163.com; wuxc@nanoctr.cn

${ }^{\dagger}$ Lin Long and Jianbo Liu contributed equally to this work

${ }^{1}$ College of Opto-electronic Engineering, Zaozhuang University, Zaozhuang 277160, China

${ }^{3}$ CAS Key Laboratory of Standardization and Measurement for Nanotechnology, National Center for Nanoscience and Technology, Beijing 100190, China

Full list of author information is available at the end of the article
} 